\title{
Experiencias obtenidas con la certificación google educator level 1
}

\section{Experiences obtained with the google educator level 1 certification}

DOI: $10.46932 / s f j d v 3 n 1-011$

Received in: Dec 30st, 2021

Accepted in: Jan 1th, 2022

Félix Molina Angel
Maestro en Ciencias, en Ciencias Computacionales
Universidad Autónoma de Guerrero,
Av. Lázaro Cárdenas, S/N, Facultad de Ingeniería, Ciudad Universitaria, Chilpancingo, Guerrero, C.P.
39087, México
E-mail: molina@ uagro.mx
Yamel Mendoza Reducindo
Estudiante de la carrera de Ingeniero en Computación
Universidad Autónoma de Guerrero
39087, México
Av. Lázaro Cárdenas , S/N, Facultad de Ingeniería, Ciudad Universitaria, Chilpancingo, Guerrero, C.P.
E-mail: merymza945@gmail.com
Valentin Alvarez Hilario
Universidad Autónoma de Guerrero
Doctor en Innovación y Administración Educativa
Av. Lázaro Cárdenas , S/N, Facultad de Ingeniería, Ciudad Universitaria, Chilpancingo, Guerrero, C.P.
39087, México
E-mail: valvarezh@uagrovirtual.mx
Iván Gallardo Bernal
Doctor en Sistemas Computacionales
Universidad Autónoma de Guerrero
E-mail: drivangallardo@ gmail.com
Av. Lázaro Cárdenas , S/N, Facultad de Ingeniería, Ciudad Universitaria, Chilpancingo, Guerrero, C.P.

\section{RESUMEN}

En el presente trabajo se promueve que los estudiantes y profesores de cualquier carrera universitaria de la Universidad Autónoma de Guerrero presenten y aprueben la Certificación de Google Educator Level 1, para asegurar el uso y aplicación correcta de las tecnologías informáticas y de comunicación utilizadas en el aula digital. Aquí se describen las funciones principales de las herramientas que abarca este programa y la experiencia adquirida en la realización del examen para lograr la certificación de este nivel.

Palabras clave: Google Workspace, Google Educator Level 1.

\section{ABSTRACT}

This paper promotes that students and professors of any university career of the Autonomous University of Guerrero present and pass the Google Educator Level 1 Certification, to ensure the correct use and 
application of computer and communication technologies used in the digital classroom. Here we describe the main functions of the tools covered by this program and the experience gained in taking the exam to achieve certification at this level.

Keywords: Google Workspace, Google Educator Level 1.

\section{INTRODUCCIÓN}

A partir de que el virus SARS-CoV2 (COVID-19) es reconocido como una enfermedad de atención prioritaria por el Consejo de Salubridad, la Secretaría de Salud publicó el acuerdo por el que se establecieron las medidas preventivas a implementar para la mitigación y control de los riesgos para la salud debido al virus COVID-19 (DOF, 2020), y ésto trajo como consecuencia que las instituciones educativas establecieran medidas emergentes para evitar la suspensión de clases. La Universidad Autónoma de Guerrero (UAGro) se apegó a los lineamientos establecidos por la Secretaría de Educación Pública, para la continuidad de actividades académicas a través de plataformas digitales (SEP, 2020).

Cabe destacar que aunque en la UAGro se promovió el uso de cuentas de correo institucionales para que profesores y estudiantes tuvieran acceso a las herramientas de tecnologías de la información de Google y así aprovechar los beneficios de las herramientas de oficina, de comunicación y aula digital, lo cierto es que una gran cantidad de estudiantes y profesores desconocían la existencia y uso de estas aplicaciones, razón por la cual se impulsó un plan de capacitación básica emergente en el uso de Google Classroom y Google Meet. Éstas y otras herramientas forman parte de Google Workspace for Education (antes GSuite).

Google Workspace for Education se compone de un conjunto de herramientas pensadas para centros educativos tradicionales y otras instituciones que imparten clases desde casa para colaborar y agilizar el aprendizaje de forma segura. Para cubrir las necesidades de una organización, Google Workspace for Education (Workspace,2021), ofrece las siguientes opciones:

- Google Workspace for Education Fundamentals: proporciona herramientas para facilitar la enseñanza y el aprendizaje, como Classroom, Google Meet, Documentos de Google, Formularios de Google y Google Chat.

- Google Workspace for Education Standard: proporciona las mismas herramientas que Education Fundamentals, pero con funciones de seguridad avanzadas y controles de administración mejorados.

- Teaching and Learning Upgrade: añade funciones mejoradas de comunicación por vídeo, complementos de Classroom y otras funciones y herramientas a tu edición Education Fundamentals o Education Standard. 
- Google Workspace for Education Plus: incluye todas las funciones de Education Standard y Teaching and Learning Upgrade, además de funciones adicionales en ciertos servicios, como el registro de asistencia de Google Meet.

Education Fundamentals es gratis para todas las instituciones que cumplan con los requisitos. Education Standard, Teaching and Learning Upgrade, y Education Plus son suscripciones de pago.

Las herramientas y funciones que ofrece Google Workspace for Education y que son incluidas en la certificación Google Educator Level 1, se describen a continuación:

- Gmail: Es considerado como un centro de trabajo potente desde el cual se pueden enviar y recibir mensajes de correo electrónico, crear espacios de trabajo y reuniones, todo desde un solo lugar. (GMail, 2021)

- Google Calendar. Con esta herramienta se puede gestionar el tiempo con calendarios en línea integrados, diseñados para el trabajo en equipo. Entre las funciones principales se incluyen: la creación y actualización de eventos, la selección de de un evento en el calendario para unirse a una invitación, unirse a una videollamada o modificar un evento, agregar o personalizar calendarios. (Google Calendar, 2021)

- Google chat. Es una plataforma de mensajería instantánea enfocada a equipos. Con Chat se puede platicar con otra persona, o establecer un espacio específico para trabajar en grupo. Con Google Chat se facilita la colaboración de forma organizada. También desde chat se pueden compartir Documentos, Hojas de Cálculo y presentaciones desde un solo lugar. (Google Chat, 2021)

- Google Meet. Es la herramienta a través de la cual se pueden realizar o programar videoconferencias desde cualquier dispositivo, unirse a clases de formación virtual, entrevistas remotas y otras tareas relacionadas. Se puede acceder a las reuniones desde una invitación creada desde el calendario, mediante código de reunión o a través de un enlace. (Google Meet, 2021)

- Google Classroom. Se utiliza para optimizar la gestión de tareas y para mejorar la colaboración y la comunicación entre profesores y estudiantes. Está disponible a través de la Web o como aplicación móvil. Algunas de las facilidades y funciones de classroom son: integración con las herramientas de oficina, Calendar, Meet, Task y otras herramientas. Desde classroom se pueden iniciar videoconferencias, gestionar clases, tareas y calificaciones, agregar cualquier tipo de materiales y compartir anuncios desde el tablero de anuncios o por correo electrónico, entre otras tareas. (Google Classroom, 2021)

- Google Drive. Es un disco duro virtual en el que se puede almacenar cualquier tipo de archivo y se pueden conceder permisos para ver o modificar a los usuarios que autorice el propietario del 
archivo. Desde Google Drive se puede subir o descargar archivos, organizar carpetas y archivos, crear y editar documentos en línea o sin conexión a Internet. (Google Drive, 2021)

- Google Docs. Es un procesador de textos en línea, a través del cual se pueden crear, editar y dar formato a documentos. Algunas de las funciones principales que se pueden hacer con un documento de este tipo son: editar y añadir estilos, trabajar con diferentes versiones y copias del documento, agregar funciones para mejorar la presentación del documento, compartir un documento para editar, comentar o leer un documento, asi como colaborar en tiempo real. (Google Docs, 2021).

- Google Sheets. Con esta aplicación se pueden crear y editar de forma simultánea hojas de cálculo. Es posible analizar datos mediante gráficos y filtros, gestionar listas de tareas y crear planes de proyectos. Algunas de las funciones más importantes son: personalización de la hoja de cálculo y datos, trabajo con filas, columnas y celdas, creación de copias y versiones diferentes de las hojas de cálculo, compartición y colaboración en tiempo real. (Google Sheets, 2021)

- Google Slides. Mediante esta herramienta, se crean y editan de manera simultánea presentaciones de proyectos, módulos de formación y otros. Las funciones básicas que se pueden realizar con esta aplicación son: creación y edición de contenido en diapositivas, organización de diapositivas, trabajo con distintas copias y versiones de la presentación, compartición y colaboración en tiempo real como editor, lector o comentador. (Google Slides, 2021)

- Google Forms. Con formularios se puede gestionar el registro de asistentes a eventos, crear cuestionarios y encuestas, analizar respuestas y otras tareas. Las funciones principales que se pueden hacer con Google Forms son: personalización, previsualización y definición de opciones para las respuestas, envío del formulario y análisis de respuestas. (Google Forms, 2021)

- Google Sites. Esta herramienta se utiliza para crear espacios web sobre proyectos internos, sitios web para equipos y sitios Web dirigidos al público. Desde Google Sites, es posible crear sitios en base a plantillas, personalizar un sitio web y la página principal, agregar páginas y seleccionar el modo de navegación, insertar contenido para cada página, revisar, compartir y publicar un sitio web. (Google Sites, 2021)

- Google Tasks. Con Tasks, se pueden crear listas de tareas pendientes, agregar fechas de vencimiento, y marcar aquellas tareas que se han completado. (Google Tasks, 2021)

Además de las herramientas descritas, el examen incluye temas básicos de Google Chrome y Youtube, dado que juegan un papel importante en la búsqueda y selección de información. 


\section{METODOLOGÍA DE DESARROLLO}

Puede haber diversas razones que justifiquen la preparación y presentación del examen de certificación Google Educator Level 1, pero en opinión de Yamel Mendoza y Félix Molina, éstas son las esenciales:

1. Muchos de los profesores de educación básica y, de nivel medio superior y superior, así como los estudiantes no cuentan con las habilidades digitales necesarias para usar correctamente las herramientas de tecnologías de la información contempladas en este programa de certificación, y las circunstancias actuales exigen que los profesores y estudiantes se familiaricen con la alfabetización digital. Ahora bien, desde la perspectiva de los estudiantes de la UAGro, esta certificación podría recomendarse que se tomara en cuenta como una opción para la acreditación de la unidad de aprendizaje "Manejo de Tecnologías de la Información", ya que garantiza el uso correcto de las herramientas de tecnologías de la Información y la Comunicación.

2. Además del uso correcto de las tecnologías señaladas, la certificación incluye temas relacionados con el trabajo en equipo, colaboración y comunicación digital, búsquedas eficientes, mejor organización, reducción del uso de papel y, el fomento a la concientización sobre el respeto, protección y navegación segura.

En base a las razones anteriores, a continuación se describen las formas de realizar la certificación siguiendo esta metodología, con algunos ajustes que se señalan: capacitación sobre aspectos básicos, videos sobre la certificación Google Educator Level 1 y presentación del examen de certificación.

\subsection{CAPACITACIÓN SOBRE ASPECTOS BÁSICOS}

Esta capacitación básica de Google consta de 13 temas: Prepárate para utilizar la tecnología en el aula, Expande tu acceso a la ayuda y al aprendizaje, Logra un aula casi libre de papel, Ahorra tiempo en la comunicación, Organiza actividades para tí y los demás, Realiza reuniones en línea, Digitaliza el trabajo de los alumnos, Mide, comprende y comparte el crecimiento de los alumnos, Enséñale a los alumnos habilidades en línea, Crea clases interactivas, Cautiva a tu clase con videos, Facilita el trabajo grupal, Fomenta la ciudadanía digital y comportamiento adecuado en línea (Skillshop, 2021). Es importante destacar que aunque cada tema pudiera parecer sencillo sobretodo para profesores y estudiantes del área de tecnologías, lo cierto es que para los estudiantes y profesores áreas ajenas a TI, la mayoría de temas le serán de bastante utilidad. Aunque Yamel es del área de TI, esto es lo que rescató del material de capacitación oficial:

“En cada parte se encontrarán detalles que uno podría desconocer, al menos yo así lo percibí y adquirí mucho conocimiento y habilidades conforme avancé en cada tópico, de tal manera que consumí más tiempo del que está establecido para cubrir todo el curso”. 
En el caso del profesor Félix Molina, ésto es lo que expresó del material de aprendizaje de Google:

"Al hacer una revisión rápida de los temas de Google Educator, me di cuenta que era necesario reforzar algunos aspectos teóricos relacionados con las habilidades de interacción y colaboración entre pares para enriquecer el conocimiento pero también para apoyar a otros docentes. Algo similar me sucedió con el uso y aplicación de algunas herramientas de Google Workspace, así que me ayudó a reforzar mis habilidades técnicas básicas de TI"

\title{
2.2 VIDEOS SOBRE LA CERTIFICACIÓN GOOGLE EDUCATOR LEVEL 1
}

Además de revisar el material principal, es altamente recomendable complementar el aprendizaje a través de videos en Youtube, ya que ésta es una forma que facilita el aprendizaje de muchas personas. Ésto es lo que expresó Yamel acerca de la capacitación con videos:

\begin{abstract}
"A mi me resultaron de mucho apoyo, estos recursos recursos: Educador Certificado de Google Nivel 1 publicado por el Instituto Jaime Torres Bodet (2020), Como pasar el examen de Google Educator Level 1-Todo sobre el examen de Educador de Google nivel 1 de acuerdo con Iriarte Cintia (2020), Curso intensivo Educadores de Google Nivel 1-Día 1 por Carreira y Gómez (2021), y Como Obtener la Certificación de Google Nivel 1-Mi experiencia para ser Google Educator por Hic Yanet (2021). Quiero enfatizar que estos videos me ayudaron a consolidar mi formación y a tener una idea más clara sobre la estructura del examen y tips para la aprobación del mismo".
\end{abstract}

\subsection{PRESENTACIÓN DEL EXAMEN}

La certificación Google Educator Level 1 tiene una vigencia de tres años contados a partir de la fecha que se aprueba el examen, este examen tiene una duración de tres horas y un costo de 10 dólares. El proceso de registro para el examen de certificación se inicia desde el sitio del Centro de Profesores (2021), desde donde se hace clic en Registrarse, y en la siguiente página se selecciona el idioma, enseguida se accede a la página de Certification Exams (2021), en la que se registran los datos del sustentante, como se ilustra en la figura 1.

Figura 1. Registro para el examen de certificación

\section{Google for Education Certification Exams}

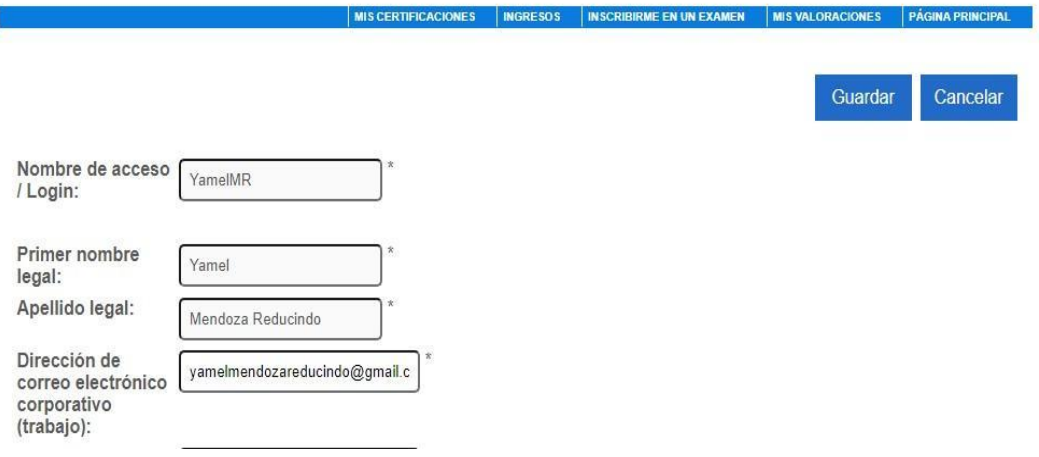


En esta misma página se accede a la pestaña "Inscribirme en un examen" desde donde se selecciona el examen y se realiza el pago. Una vez completado este proceso, el sistema envía los datos de acceso, periodo durante el cual estará disponible el examen y demás indicaciones para la realización del mismo.

Al iniciar el examen, el sistema informa que éste consta de 20 preguntas de conocimientos de opción múltiple y, de arrastrar y soltar, así como 11 casos prácticos, cada uno de los cuales incluye dos o más tareas a realizar siguiendo las instrucciones que correspondan. Otra información que puede tomarse en cuenta es que durante el examen se puede consultar cualquier recurso disponible en línea relacionado con el examen, pero queda estrictamente prohibido preguntarle a otra persona.

Respecto a la experiencia de presentación del examen de certificación, ésto es lo que describe el profesor Félix Molina:

\begin{abstract}
“Después de una preparación rápida por aproximadamente quince días, decidí registrarme para presentar el examen de Google Educator Level 1, en idioma Inglés. Y tomando en cuenta las recomendaciones ya leídas le dediqué justo alrededor de 30 minutos a la sección teórica de 20 preguntas, y el tiempo restante (150 minutos) a la sección de 11 casos prácticos. Quiero compartir que al principio pensé que no iba a necesitar todo el tiempo asignado pero lo cierto es solo logré completar nueve casos. Afortunadamente, logré acreditar el examen en el primer intento"
\end{abstract}

Por su parte, Yamel Mendoza, relata así su experiencia respecto a la presentación del examen de certificación:

\begin{abstract}
"Es importante relatar que en mi caso presenté el examen en tres ocasiones: En el primer intento se me presentaron en la primera parte, preguntas de razonamiento de opción múltiple y otras de arrastrar y soltar. Como primera impresión pude percibir que las preguntas no eran difíciles pero si confusas y en algunos casos percibidas como fuera de contexto debido a que algunas frases no estaban correctamente traducidas al español y/o a que otras palabras no estaban traducidas. Esto pudo afectarme en el tiempo pero sobre todo en el resultado. En el apartado de los casos prácticos creo que no estuvieron complicados pero si me tomaron tiempo, de modo que no alcancé a resolver dos de los 11 casos. En este primer intento alcancé $72 \%$ del $80 \%$ necesario para aprobarlo, y de acuerdo a la retroalimentación recibida tuve fallas relacionadas con búsquedas en GMail y con el uso de Google Sites para la creación de sitios Web. Así que decidí programar el segundo intento a los quince días.En este segundo intento, el examen consistió de 37 preguntas teóricas y ningún caso práctico, pero tal vez confié demasiado en mi misma, de modo que lo terminé en poco tiempo pero el resultado fué el mismo y con la misma retroalimentación. Ésto me puso a recapacitar sobre el tipo de preguntas, de modo que preferí dar un buen repaso a los cuestionarios de cada tema del programa de capacitación, y programé mi tercer intento a los 60 días tal como lo establece Google. En este tercer y último intento el examen fué presentado sólo con 37 preguntas teóricas, pero como ya había retomado en serio la retroalimentación, logré finalmente aprobarlo".
\end{abstract}

Es esencial mencionar, que cuando se consigue acreditar el examen de certificación, no se hace mención al puntaje alcanzado. 


\section{COMENTARIOS FINALES}

En opinión de Yamel Mendoza y de Félix Molina, aún cuando pueda haber uno o más intentos fallidos en el examen, la presentación del examen representa una experiencia muy valiosa para el fortalecimiento de la formación profesional, y aunque podrían percibirse como tecnologías básicas, vale la pena validar nuestros conocimientos y habilidades a través de esta certificación.

\section{RESUMEN DE RESULTADOS}

En este trabajo se describieron las aplicaciones y funciones de las herramientas que forman parte del programa Google Workspace for Education, el proceso de preparación recomendado para la presentación del examen de certificación, la experiencia obtenida durante proceso de capacitación y en los momentos de realización del examen, el resultado del examen y la retroalimentación proveída por Google..

\section{CONCLUSIONES}

Aunque se trata de un examen relacionado con las herramientas básicas de Google, siempre será necesario realizar un repaso acorde al tipo del participante, considerando los aspectos teóricos y prácticos poniendo especial énfasis en la aplicación y uso correcto de las aplicaciones. Esto quiere decir que siempre es mejor repasar más de una vez el área de conocimientos y desarrollar las habilidades técnicas tomando en cuenta que un problema puede resolverse con diferentes alternativas, con la finalidad de conseguir un resultado satisfactorio desde el primer intento.

\section{RECOMENDACIONES}

Para quienes estén interesados en tomar este examen de certificación, se les recomienda repasar muy bien cada tema, realizar suficiente práctica enfocada solo a los tópicos vistos y a su integración con las demás herramientas, y que administren adecuadamente el tiempo, estableciendo en la medida de lo posible no más de 30 minutos para la parte teórica y el resto para los casos prácticos. 


\section{REFERENCIAS}

Ayuda de Classroom. Acerca de Classroom. Centro de Asistencia. Consultado por Internet, el 20 de septiembre de 2021. Dirección de Internet:

https://support.google.com/edu/classroom/answer/6020279?hl=es\&ref_topic=7175444

Centro de Profesores. Obtén la Certificación de Educador de Nivel 1. Google for Education, 2021. Consultado por Internet el 5 de julio de 2021. Dirección de Internet: https://edu.google.com/intl/ALL_mx/teacher-center/certifications/educator-level1/?modal_active=none

Carreira, Belén y Gómez, Mauricio. Curso Intensivo Educadores Google Nivel 1-Día 1. GEG Buenos Aires-GEG Tucumán, Argentina 2021. Consultado por Internet, el 29 de junio de 2021. Dirección de Internet: https://www.youtube.com/watch?v=Uiu0sZNbyyM\&t=2009s

Centro de aprendizaje de Google Workspace. Formación y ayuda sobre GMail. Centro de Asistencia. Consultado por Internet, el 20 de septiembre de 2021. Dirección de Internet: https://support.google.com/a/users/answer/9259748?visit_id=637707225554950861-

991844878\&hl=es\&rd=1

Centro de aprendizaje de Google Workspace. Hoja resúmen de Google Chat. Centro de Asistencia. Consultado por Internet, el 20 de septiembre de 2021. Dirección de Internet: https://support.google.com/a/users/answer/9299928

Centro de aprendizaje de Google Workspace. Hoja resúmen de Calendar. Centro de Asistencia. Consultado por Internet, el 20 de septiembre de 2021. Dirección de Internet: https://support.google.com/a/users/answer/9290350

Centro de aprendizaje de Google Workspace. Hoja resúmen de Google Meet. Centro de Asistencia. Consultado por Internet, el 20 de septiembre de 2021. Dirección de Internet: https://support.google.com/a/users/answer/9300131

Centro de aprendizaje de Google Workspace. Hoja resúmen de Documentos. Centro de Asistencia. Consultado por Internet, el 20 de septiembre de 2021. Dirección de Internet: https://support.google.com/a/users/answer/9299931

Centro de aprendizaje de Google Workspace. Hoja resúmen de Hojas de Cálculo. Centro de Asistencia. Consultado por Internet, el 20 de septiembre de 2021. Dirección de Internet: https://support.google.com/a/users/answer/9300022

Centro de aprendizaje de Google Workspace. Hoja resúmen de Presentaciones. Centro de Asistencia. Consultado por Internet, el 20 de septiembre de 2021. Dirección de Internet: https://support.google.com/a/users/answer/9300133

Centro de aprendizaje de Google Workspace. Hoja resúmen de Formularios. Centro de Asistencia. Consultado por Internet, el 20 de septiembre de 2021. Dirección de Internet: https://support.google.com/a/users/answer/9299716

Centro de aprendizaje de Google Workspace. Hoja resúmen de Sites. Centro de Asistencia. Consultado por Internet, el 20 de septiembre de 2021. Dirección de Internet: https://support.google.com/a/users/answer/9300023 
Centro de aprendizaje de Google Workspace. Formación y ayuda sobre Google Tasks. Aprendizaje por Producto. Consultado por Internet, el 20 de septiembre de 2021. Dirección de Internet: https://support.google.com/a/users/answer/9991367

DOF. Diario Oficial de la Federación (24/03/2020). ACUERDO por el que se establecen las medidas preventivas que se deberán implementar para la mitigación y control de los riesgos para la salud que implica la enfermedad por el virus SARS-CoV2 (COVID-19). Consultado por Internet, el 20 de septiembre de 2021. Dirección Internet: https://www.dof.gob.mx/nota_detalle.php?codigo=5590339\&fecha=24/03/2020

Google for Education. Capacitación sobre aspectos básicos. Skillshop. Consultado por Internet el 17 de mayo de 2021. Dirección de Internet: https://skillshop.exceedlms.com/student/path/111629-capacitacionsobre-aspectos-basicos

Hic, Yanet. Como Obtener la Certificación de Google Nivel 1-Mi experiencia para ser Google Educator. 6 de enerro de 2021. Consultado por Internet, el 2 de julio 2021. Dirección de Internet:https://www.youtube.com/watch?v=9HX88rnTgJM\&t=4s

Instituto Jaime Torres Bodet. Educador Certificado de Google Nivel 1. Instituto Jaime Torres Bodet. 28 de Junio de 2020. Consultado por Internet, el 24 de junio de 2021. Dirección de Internet: https://www.youtube.com/watch?v=n1A1ExnKEIg

Iriarte, Cintia. Cómo pasar el examen de Google Educator Level 1-Todo sobre el examen de Educador de Google nivel 1 de acuerdo con Cintia Iriarte. Septiembre de, 2020. Consultado por Internet el 27 de junio de 2021. Dirección de Internet: https://www.youtube.com/watch?v=P0YGmJ5-2rw\&t=12s

Subsecretaría de Educación Superior. LINEAMIENTOS DE ACCIÓN COVID-19 INSTITUCIONES PÚBLICAS DE EDUCACIÓN SUPERIOR. Secretaría de Educación Pública, 14 de marzo de 2020. Consultado por Internet el 20 de septiembre de 2020. Dirección de Internet: http://www.anuies.mx/recursos/pdf/LINEAMIENTOS_COVID-19_IES_SES_VFINAL.pdf

Webassesor. Certifications Exams. Google for Education, 2021. Consultado por Internet el 8 de julio de 2021.Dirección Internet: https://www.webassessor.com/wa.do?page=editSelfCandidate $\&$ selectedFR=CANDIDATE $\&$ tabs $=1$

Workspace. Descripción general de Google Workspace for Education. Ayuda de Administrador de Google Workspace, Centro de Asistencia. Consultado por Internet, el 20 de septiembre de 2021. Dirección de Internet:

https://support.google.com/a/answer/7370133?hl=es\&ref_topic=3035696 